\title{
Production of Polyploid Meth-A Cells Whose Ploidy is not a Power of 2 by Staurosporine
}

\author{
Kohzaburo Fujikawa-Yamamoto*, Hiroko Yamagishi and Minoru Miyagoshi \\ Division of Cell Medicine, Research Institute of Medical Science, Kanazawa Medical University, \\ Uchinada, Ishikawa 920-0293, Japan
}

Received July 13, 2004; accepted July 22, 2004

\begin{abstract}
Summary To examine ploidy change in hyperploid cells after treatments of staurosporine (SS), an inhibitor of protein kinases, octaploid Meth-A cells were exposed to SS, and then the drug concentration was gradually reduced. Seventy cell clones were obtained from the cell population, and they were classified into 4 groups based on their DNA content corresponding to hexa-, octa-, dodeca- and hexadecaploid cells. Durations of $\mathrm{G}_{1}, \mathrm{~S}$ and $\mathrm{G}_{2} / \mathrm{M}$ phases of the cells were almost the same as that of parent octaploid cells. The cell volumes of 6- and 12-ploid cells were not proportional to DNA content. It was concluded that polyploid Meth-A cells whose ploidy is not a power of 2 were produced from octaploid cells by SS treatments.

Key words Meth-A cells, Octaploid cells, Hexaploid cells, Dodecaploid cells, Hexadecaploid cells, Staurosporine.
\end{abstract}

Meth-A cells (a methylcholanthrene-induced mouse abdominal dropsy sarcoma cell line) always contain a small population of large cells that are produced from diploid cells by spontaneous polyploidization and removed eventually by apoptosis (Fujikawa-Yamamoto et al. 1997a). Meth-A cells may be susceptible to polyploid transformation. Several important characteristics of polyploidized Meth-A cells have been studied, including DNA synthesis (Fujikawa-Yamamoto et al. 1997b), apoptosis (Fujikawa-Yamamoto et al. 1997a, Zong et al. 1998), growth in vivo (Zong et al. 1998), cell surface hydrocarbon chains (Fujikawa-Yamamoto et al. 2000) and involvement of protein kinase C (Zong et al. 1995, Zong et al. 2000).

A tetraploid Meth-A cell line was established from diploid Meth-A cells highly polyploidized by demecolcine (Fujikawa-Yamamoto et al. 2001). An octaploid Meth-A cell line was also established through transient hexadecaploid cells polyploidized by demecolcine (Fujikawa-Yamamoto et al. 2003). It seems that polyploidization by demecolcine produces polyploid Meth-A cells whose ploidy is the power of 2 . While di-, tetra- and octaploid Meth-A cell lines may provide a cell system for investigating the alterations induced by polyploidization, it is not clear whether or not the polyploid Meth-A cells whose ploidy is not a power of 2 can be produced.

Staurosporine (SS), a natural alkaloid discovered from culture broth of Streptomyces staurosporeus, is a potent inhibitor of protein kinases, including C and A (Zollner 1993). SS exerts its biological activities by competing with the binding of ATP to the kinase catalytic domain; however, the polyploidization-related enzymes that are specifically inhibited by this drug are still unknown (Tamaoki et al. 1986, Fallon 1990). We previously established a triploid V79 cell line through polyploidization by K-252a, which has a similar molecular structure to SS (Fujikawa-Yamamoto et al. 2002). Though SS can polyploidize Meth-A cells, its potential for polyploidization is less than that of K-252a (Fujikawa-Yamamoto et al. 2001).

In order to obtain Meth-A cells of ploidy other than a power of 2, we used octaploid Meth-A cells as parental cells and SS as a polyploidizing agent. In this study, several polyploid Meth-A cells

\footnotetext{
* Corresponding author, e-mail: fujikawa@kanazawa-med.ac.jp
} 
having DNA content corresponding to hexa- (6-), dodeca- (12-) and hexadecaploid (16-ploid) were obtained and examined regarding cell cycle, cell volume and morphology.

Materials and methods

Cells

Octaploid Meth-A cells were maintained in a humidified atmosphere of $5 \% \mathrm{CO}_{2}$ at $37^{\circ} \mathrm{C}$ as a suspension culture in a Leibovitz's L15: Ham's F10 mixture $(7: 3)$ supplemented with $10 \%$ fetal bovine serum (CELLect GOLD, ICN Biomedicals, Aurora, OH, USA), streptomycin $(100 \mu \mathrm{g} / \mathrm{ml})$ and penicillin (50 units $/ \mathrm{ml}$ ). The other polyploid Meth-A cells were cultured under the same conditions described above.

\section{Drug addition and drug removal}

Exponentially growing octaploid Meth-A cells were plated in culture flasks $\left(25 \mathrm{~cm}^{2}\right.$, Corning Costar Co., Acton, MA, USA) at a density of about $1 \times 10^{6}$ cells/flask. Twelve hours later, the cells were exposed to staurosporine (SS, $100 \mathrm{nM}$, Funakoshi, Tokyo, Japan) for 24 or $48 \mathrm{~h}$. Then, the drug concentration of the culture flasks was reduced one half by a $1: 2$ dilution with fresh medium. This procedure of concentration reduction was repeated every day for $6 \mathrm{~d}$. Then, the Meth-A cells were subcultured and cellular DNA content was checked by flow cytometry (FCM).

\section{Cell cloning}

The Meth-A cells $22 \mathrm{~d}$ after SS addition were seeded in 96-well dishes (96-well tissue culture clusters, 6.4-mm diameter, Costar Co., Cambridge MA, USA) at low density. The wells containing

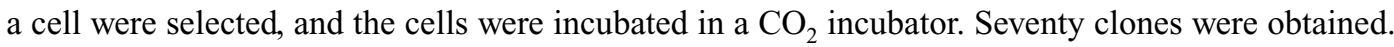
At $18 \mathrm{~d}$ after cloning, the first FCM measurement for DNA histograms was performed to check the cellular DNA content of cells. At that time, the expected number of cell divisions from the first cloning was greater than 20. Hereafter, the cells obtained will be called n-ploid Meth-A cells for convenience, though in some cases the ploidy has not been confirmed by karyotyping.

\section{Cell growth}

The 6-, 8-, 12- and 16-ploid Meth-A cells were subcultured every day by $1: 2$ dilution in culture flasks $\left(25 \mathrm{~cm}^{2}\right.$, Corning Costar Co. $)$, and the cell number was counted using a hemocytometer at the time of subculturing. Diploid Meth-A cells were prepared as a control for the measurements. The measurements were finished within 2 months after the cell cloning.

\section{FCM measurements}

The cells obtained through the experiments were fixed with $20 \%$ ethanol, resuspended in $\mathrm{PBS}^{(-)}$(divalent cation-free phosphate-buffered saline) containing 0.25\% RNase (Type II-A, Sigma, St. Louis, MO, USA) and incubated for $1 \mathrm{~h}$ at room temperature. The cells were counted using a hemocytometer, and aliquots of about $2 \times 10^{5}$ cells were used for FCM. Immediately before the measurements, the cells were stained with propidium iodide $\left(\mathrm{PI}, 7.5 \times 10^{-5} \mathrm{M}\right)$ and examined for red fluorescence by FCM. Under these staining conditions, the signal due to residual double-stranded RNA is negligible and the relative intensity of the red fluorescence corresponds to the DNA content (Krishan 1975).

The fluorescence from individual cells was measured using a FACSORT (Becton Dickinson Immunocytometry Systems, Franklin Lake, ND, USA). The fluorescence of individual cells irradiated with a focused laser light at a wavelength of $488 \mathrm{~nm}$ was detected using a photomultiplier tube. The relative intensity of red fluorescence (FL2A and FL2H) was measured and DNA histograms were obtained. 


\section{Cell cycle analysis}

FCM data of FL2H (signals of red-fluorescence intensity through a logarithmic amplifier) for 10000 cells were input to CASL software for cell cycle analysis of DNA histograms on a log scale using the transfer software "FACS to ASCII" (freeware), and the DNA histograms were decomposed to cell fractions based on DNA content (Fujikawa-Yamamoto 1999). CASL was written using Mathematica (Ver. 2.2) and can analyze DNA histograms with a DNA content of $1 \mathrm{c}$ to $128 \mathrm{c}$. The algorithm is similar to Fried's method (Fried 1977) except that normal distribution functions having the same half-width instead of the same CV (coefficient of variation) value are used as components.

\section{Chromosome analysis}

Exponentially growing 6-, 12- and 16-ploid Meth-A cells in culture dishes $(60 \mathrm{~mm}$ diameter, Nalge Nunc International, IL, USA) were exposed to demecolcine at a concentration of $270 \mathrm{nM}$ for $1 \mathrm{~h}$. The cells were centrifuged, swelled with $75 \mathrm{mM} \mathrm{KCl}$, fixed with a fixing solution $\left(\mathrm{CH}_{3} \mathrm{OH}: \mathrm{CH}_{3} \mathrm{COOH}=7: 3\right)$ and dropped onto glass slides. The cells were stained with Giemsa solution in order to photograph the chromosomes. Chromosome numbers were counted from the photographs. Karyotype analysis was performed manually for 6-ploid Meth-A cells.

\section{Cell morphology}

The morphology of exponentially growing 2-, 6-, 8-, 12- and 16-ploid Meth-A cells in culture flasks was photographed under a low-phase contrast microscope (CK2, Olympus, Tokyo, Japan) equipped with a digital camera system (DS4040, Olympus). Then, the cells were washed once with $\mathrm{PBS}^{(-)}$, fixed with methanol, and stained with hematoxylin/eosin using the standard method. Photographs were taken under a microscope (BX 60, Olympus) equipped with a digital camera system (DS4040, Olympus). The images were entered into a personal computer and printed at the magnification stated in the text.

\section{Cell volume distribution}

Exponentially growing 2-, 6-, 8-, 12- and 16-ploid Meth-A cells were fixed with 20\% ethanol and resuspended in $\mathrm{PBS}^{(-)}$. The distribution of cell volume (Coulter volume) was measured using a Coulter Counter (ZM/256, Coulter Electronics, Fullerton, CA, USA).

\section{Results}

To examine the polyploidization of octaploid Meth-A cells by SS, the cells were exposed to SS for 1 or $2 \mathrm{~d}$. Then the concentration of drug was gradually reduced to half by subculturing daily. Changes in DNA histograms and in cell number are shown in Fig. 1. The DNA content of the main peak was $16 \mathrm{c}$ at $2 \mathrm{~d}$ after the drug addition. Meth-A cells having greater than 16c DNA content appeared at the reduced drug concentration. It was suggested that octaploid Meth-A cells were arrested in the $G_{1}$ phase of hexadecaploidized cells during SS-exposure and began to progress the cell cycle after the SS-release. It should be noted that the cell number was not increased in this period. Because the cell population following $2 \mathrm{~d}$ drug-exposure and $21 \mathrm{~d}$ after starting the drug removal was a mixture of polyploid cells, it was cloned (right panel of Fig. 1).

Seventy clones were obtained from the cloning. The ploidy of cloned cells was tentatively determined from the DNA histograms (data not shown). The DNA contents of cell populations of 2, 57, 1 and 10 clones corresponded to those of 6-, 8-, 12- and 16-ploid cells, respectively, suggesting that the ploidy transitions of 8-6 and 8-12 were rare events compared to the 8-16 transition. Figure 2 shows the DNA histograms and the changes in cell numbers during long-term culturing of polyploid Meth-A cells within 2 months after the first cloning. The doubling times of these polyploid cells were almost the same regardless of the DNA content, within the experimental error (Table 1). 

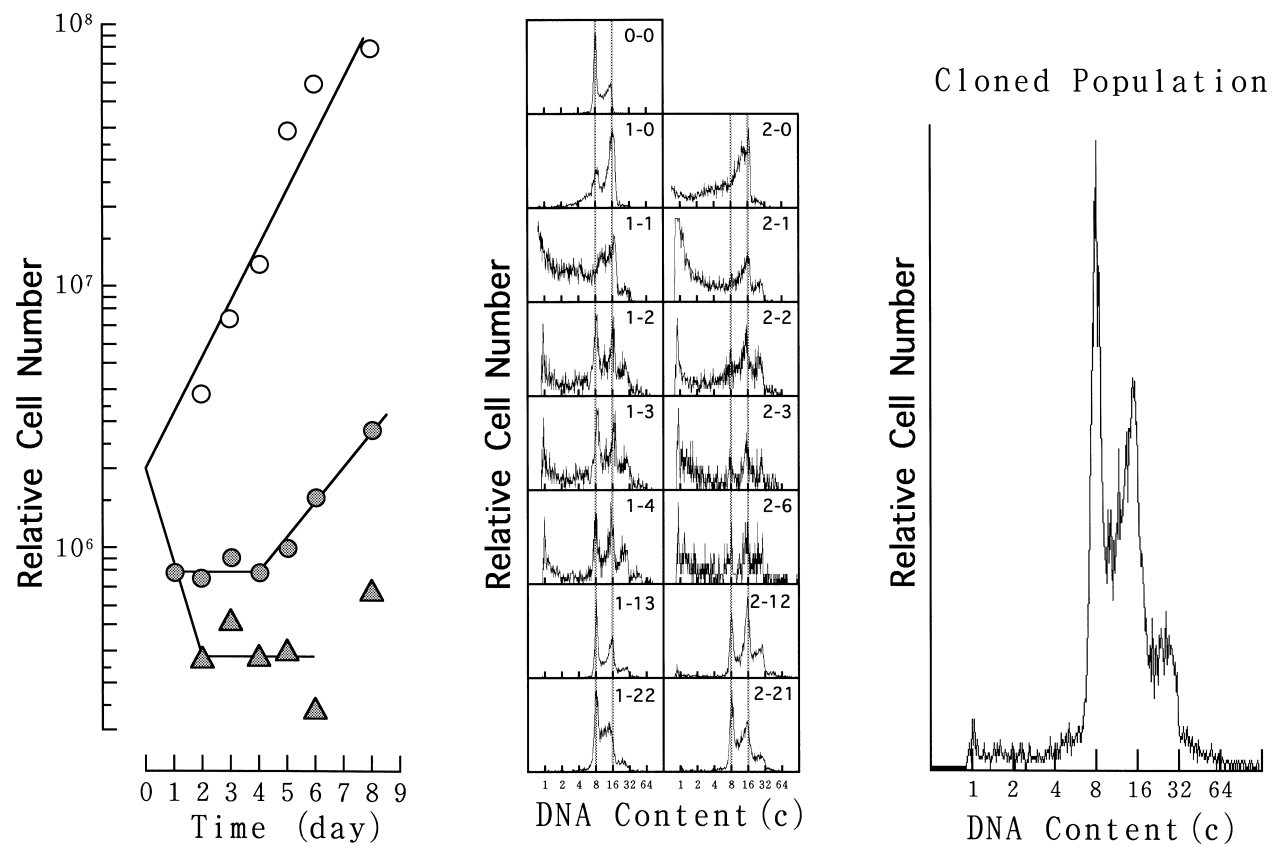

Fig. 1. Changes in cell number (left) and DNA fluorescence histograms (middle and right) of Meth-A cells after the addition and removal of staurosporine. Exponentially growing octaploid Meth-A cells were exposed to staurosporine at a concentration of $100 \mathrm{nM}$ for 1 or $2 \mathrm{~d}$. The concentration of the drug was reduced by half every. In the left panel, open circles, closed circles and closed triangles represent 0 , 1 and $2 \mathrm{~d}$ treatment with staurosporine, respectively. In the middle panel, paired numerals represent the time (days) of the drug removal and the time after removal, in that order. The right panel represents an enlarged histogram of 2-21, whose cell population was cloned. The abscissa represents the DNA content (c, complement).

To examine the integrity of 6-ploid Meth-A cells, the distribution of chromosome numbers and the karyotyping chart were examined (Fig. 3). The chromosomes numbered 125-135, proving that the cells were hexaploid. However, we could not assign the chromosomes for hexaploid Meth-A cells, because chromosomes of diploid Meth-A cells have not been assigned. The chromosomes of 12- and 16-ploid Meth-A cells numbered 150-328 and 170-353, respectively; however, karyotyping of these cells has not been done.

Representative outputs of CASL for DNA histograms of exponentially growing 2-, 6-, 8-, 12and 16-ploid Meth-A cells are shown in Fig. 4 (left panel). The phase fractions obtained using CASL and the calculated durations of $\mathrm{G}_{1}, \mathrm{~S}$ and $\mathrm{G}_{2}+\mathrm{M}$ phases are listed in Table 1. The durations of the S phase were about the same in 6-, 8-, 12- and 16-ploid Meth-A cells, implying that the rate of DNA synthesis was proportional to DNA content. Marked alterations in the phase duration did not occur in these polyploid Meth-A cells.

To examine the morphology of 2-, 6-, 8-, 12- and 16-ploid Meth-A cells, the cells were HEstained (right panel of Fig. 4). Most cells were mononuclear and exhibited normal cell division. Phase contrast micrographs of the cells in the growing state are shown in Fig. 5 (right panel). The morphology of these cells was not the same, suggesting that some functional alteration occurs with the ploidy transition.

In order to examine the production mechanism of 6-, 12- and 16-ploid cells, the cell-volume of the polyploid cells was measured within 2 months after the first cloning (left panel of Fig. 5). The ratios of cell volume of $2-: 6-: 8-: 12-: 16$-ploid cells were about $1: 6: 4: 12: 8$, suggesting that 6 and 12-ploid cells are different from 16-ploid cells in the production mechanism. 

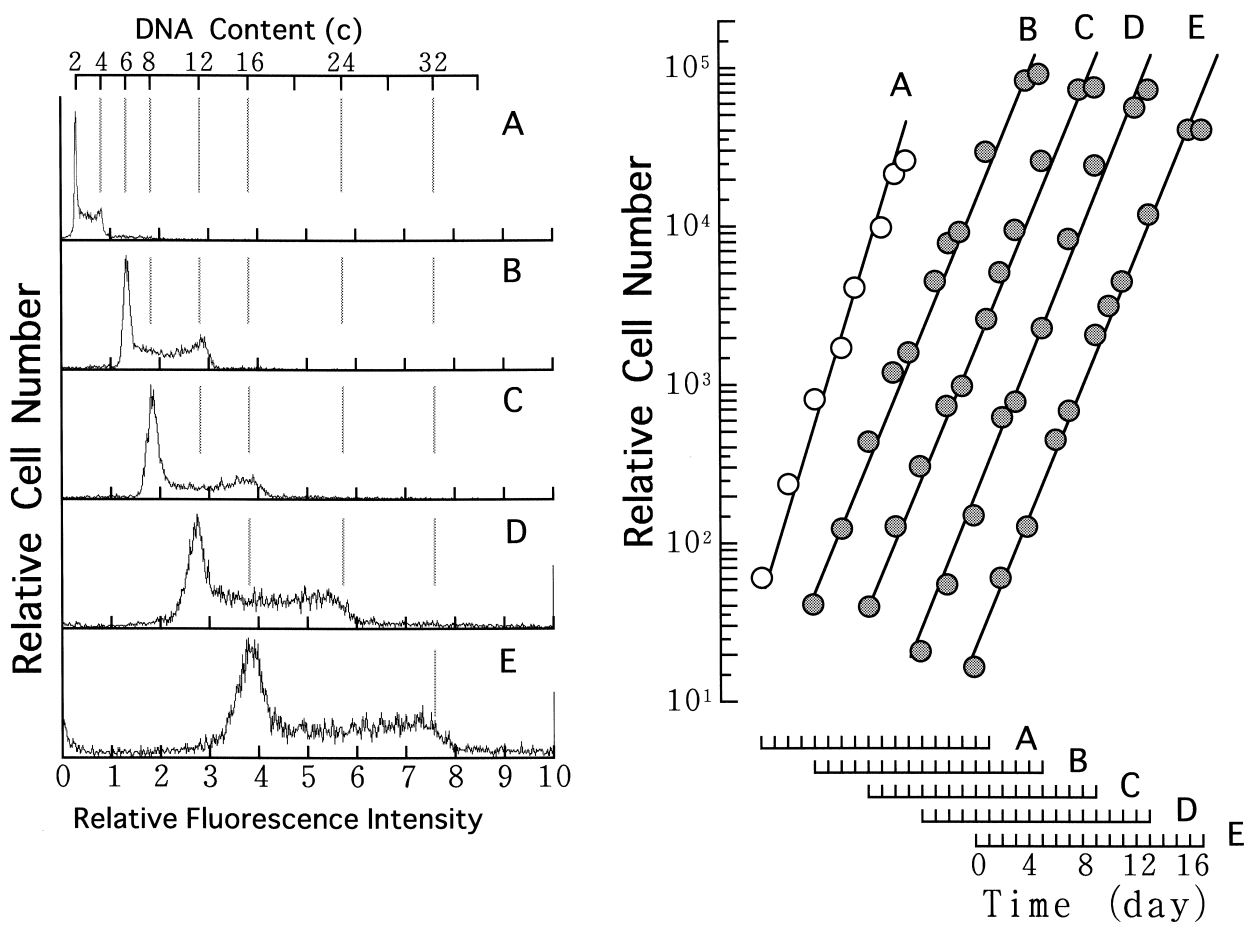

Fig. 2. DNA histograms (left panel) and growth curves (right panel) of exponentially growing 2- (A), 6- (B), 8- (C), 12- (D) and 16-ploid Meth-A cells (E) within 2 months after the first cloning. These histograms were measured under the same experimental conditions on the same day, with the same gain and with a relatively small number of cells. The abscissa represents the relative red-fluorescence intensity. Longitudinal bars and scale of DNA content are shown to facilitate understanding. In the right panel, changes in the relative numbers of 2-, 6-, 8-, 12- and 16-ploid Meth-A cells were enumerated through daily subculturing. The abscissa represents the time (days). Solid lines were drawn to facilitate understanding.

\section{Discussion}

Polyploidization of mammalian cells occurs in various organs, particularly in the aged or partially hepatectomized liver; however, the mechanisms of polyploidization are poorly understood (Mossin et al. 1994, Zong et al. 1994, Fogt and Nanji 1996, Seglen 1997). We established tetraploid and octaploid Meth-A cell lines from diploid and tetraploid Meth-A cells, respectively, that had been highly polyploidized by demecolcine (Fujikawa-Yamamoto et al. 2001, 2003a). Triploid V79 cells were also established from transient tetraploid cells polyploidized by K-252a, a protein kinase inhibitor with a molecular structure similar to SS (Fujikawa-Yamamoto et al. 2002). SS was used in this study in order to produce polyploid cells whose ploidy is not a power of 2 .

It is of interest that 6- and 12-ploid Meth-A cells were produced from octaploid cells. So long as it is assumed that polyploid cells are produced by DNA duplication without cell division, it is difficult to explain the production of these polyploid Meth-A cells from the octaploid parental cells. If the cell volume reflects the mechanisms of production of these polyploid cells, the 16-ploid cells may have been produced with DNA replication without cell division, because the cell volume was double that of the 8-ploid cells. Hexaploid cells may have been produced from 12-ploid cells through four-cell division or successive cell division, because their cell volume was half that of the 12-ploid cells. The mechanism of production of 12-ploid Meth-A cells may have been complex. Presently, we have no evidence to explain these experimental findings. 


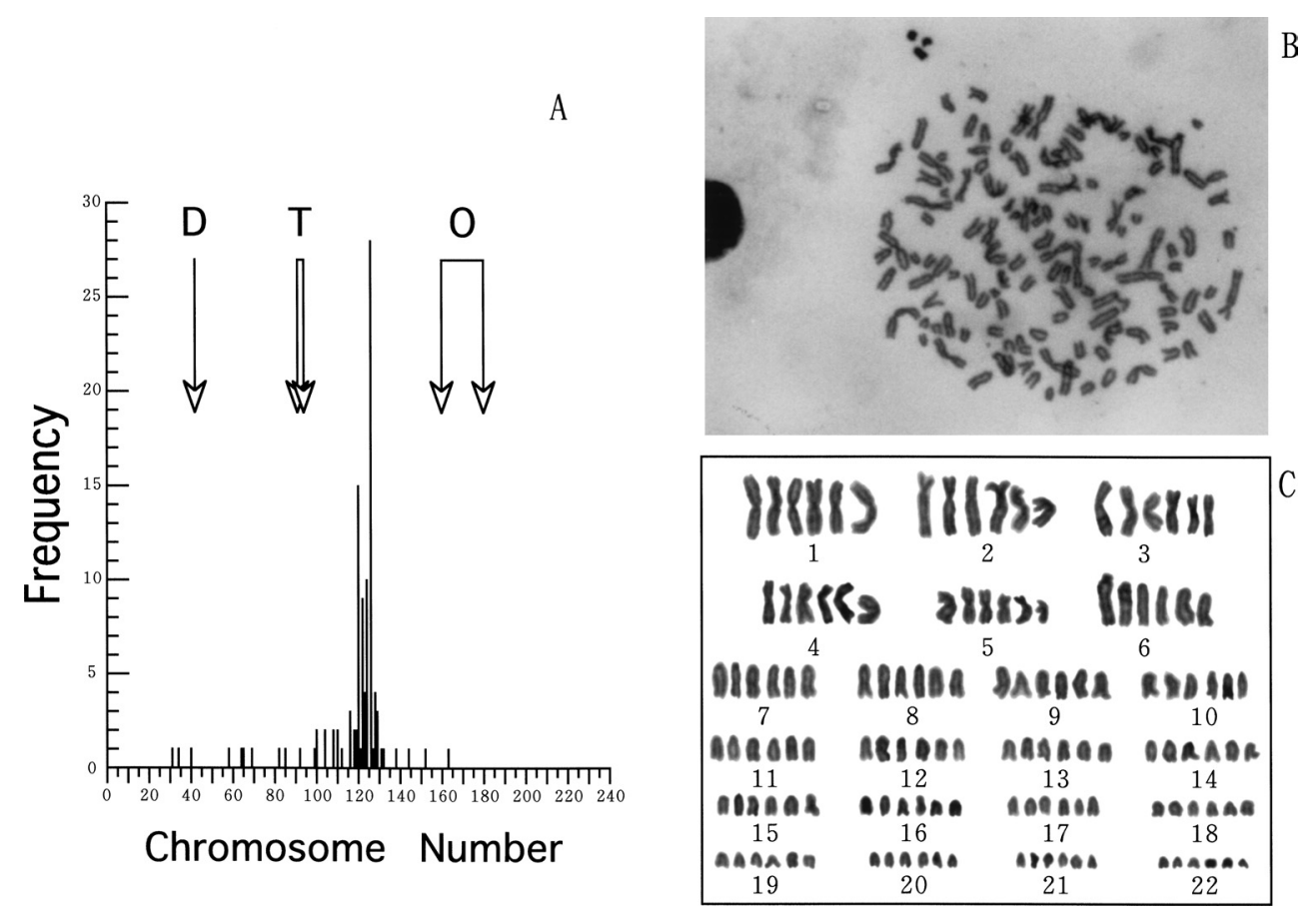

Fig. 3. Histograms of chromosome number (A), a photomicrograph (B), and a karyotyping chart (C) of 6-ploid Meth-A cells. Exponentially growing 6-ploid Meth-A cells were exposed to demecolcine at a concentration of $270 \mathrm{nM}$ for $1 \mathrm{~h}$. The chromosomes were stained with Giemsa solution. The chromosomes of about 100 cells were enumerated from enlarged photographs. For karyotyping, the photomicrograph (B) was used and the assignment of chromosomes remained obscure. Arrows, D, T and O, represent width between the 1st and 2nd peaks in the distribution histograms of 2-, 4- and 8-ploid Meth-A cells, respectively (Fujikawa-Yamamoto et al. 2001, 2003a).

Table 1. Cell cycle parameters of polyploid Meth-A cells

\begin{tabular}{|c|c|c|c|}
\hline & $\mathrm{G}_{1}$ & S & $\mathrm{G}_{2} / \mathrm{M}$ \\
\hline \multicolumn{4}{|c|}{ 2-ploid cells $(\mathrm{Td}=28 \mathrm{~h})^{*}$} \\
\hline fraction (h) & $0.281(0.245)$ & $0.561(0.490)$ & $0.158(0.138)$ \\
\hline duration $(\mathrm{h}) * *$ & 6.1 & 16.0 & 5.9 \\
\hline \multicolumn{4}{|c|}{ 6-ploid cells $(\mathrm{Td}=37 \mathrm{~h})$} \\
\hline fraction $(\mathrm{h})$ & $0.429(0.340)$ & $0.402(0.319)$ & $0.169(0.134)$ \\
\hline duration (h) & 12.9 & 15.8 & 8.3 \\
\hline \multicolumn{4}{|c|}{ 8-ploid cells $(\mathrm{Td}=37 \mathrm{~h})$} \\
\hline fraction $(\mathrm{h})$ & $0.509(0.440)$ & $0.350(0.302)$ & $0.141(0.122)$ \\
\hline duration (h) & 15.7 & 14.3 & 7.0 \\
\hline \multicolumn{4}{|c|}{ 12-ploid cells $(\mathrm{Td}=34 \mathrm{~h})$} \\
\hline fraction $(h)$ & $0.340(0.251)$ & $0.484(0.357)$ & $0.176(0.130)$ \\
\hline duration (h) & 9.1 & 16.9 & 8.0 \\
\hline \multicolumn{4}{|c|}{ 16-ploid cells $(\mathrm{Td}=36 \mathrm{~h})$} \\
\hline fraction (h) & $0.335(0.235)$ & $0.488(0.342)$ & $0.177(0.124)$ \\
\hline duration (h) & 9.5 & 18.0 & 8.5 \\
\hline
\end{tabular}

* Td is the doubling time calculated from Fig. 2. Phase fractions of polyploid Meth-A cells were determined, omitting those for cells with other ploidy in the Meth-A cell population. Numbers in parentheses represent the fraction of the total cell population.

** Phase duration was calculated using conventional equations (Watanabe and Okada 1967) employing the doubling time instead of the cycle time. 

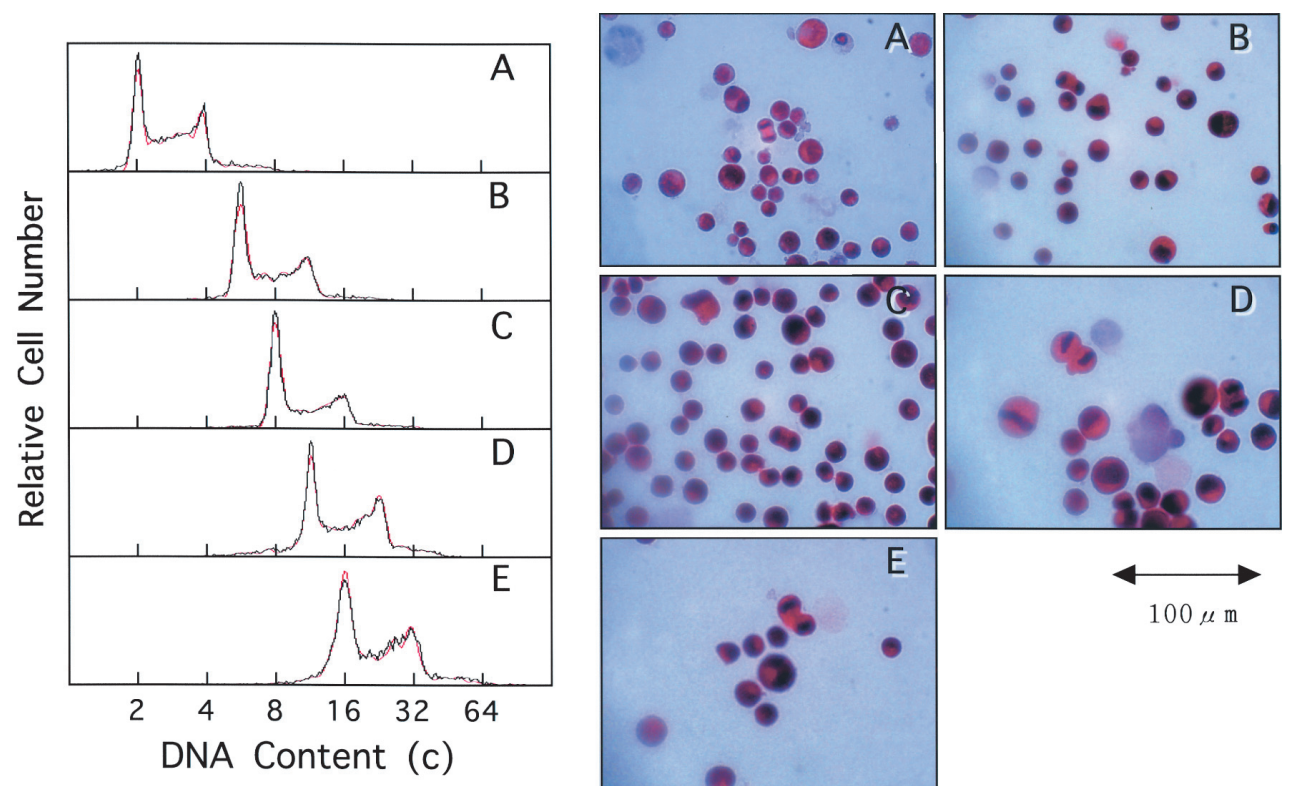

Fig. 4. Representative CASL outputs (left panel) and light micrographs (right panel) of exponentially growing 2- (A), 6- (B), 8- (C), 12- (D) and 16-ploid (E) Meth-A cells. In the left panel, solid (black) and dotted (red) lines represent experimental and synthesized histograms, respectively. In the right panel, the cells were stained with $\mathrm{HE}$.
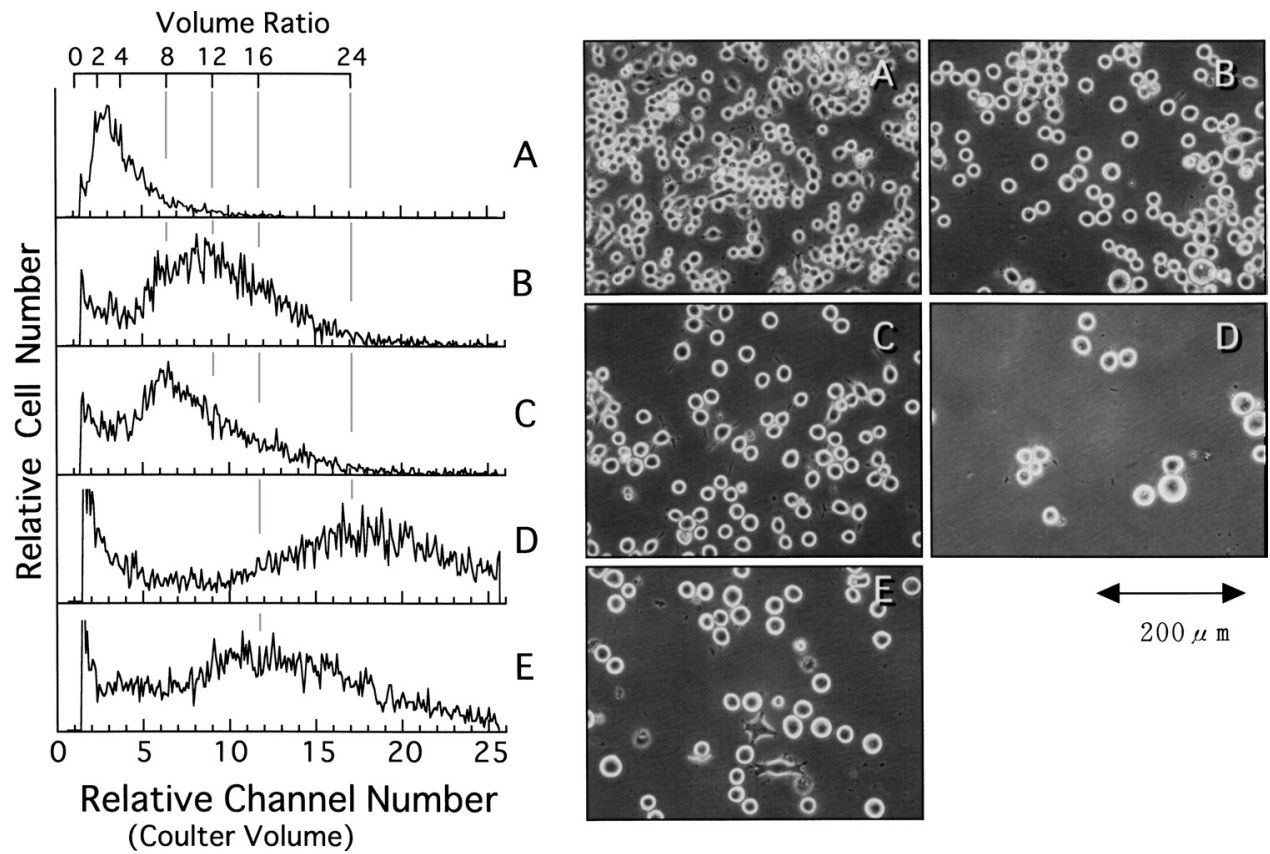

Fig. 5. Volume distribution (left panels) and phase-contrast micrographs (right panels) of exponentially growing 2- (A), 6- (B), 8- (C), 12- (D) and 16-ploid (E) Meth-A cells. In the left panel, the cell volumes were measured using a Coulter counter. Vertical lines and a scale were drawn to facilitate understanding. 
We have presented a model for the DNA structure of cells to explain the production of triploid cells from diploid V79 cells (Fujikawa-Yamamoto et al. 2002). The model suggests that DNA replication of paired double-strand DNA may be bypassed, resulting in ploidy degradation through cell division (Fujikawa-Yamamoto et al. 2003), and the cell volume will then become larger than that expected from the ploidy. SS may produce the 12-ploid Meth-A cells through the mechanism.

It is of interest that the duration of the $\mathrm{S}$ phase was almost the same in 6-, 8-, 12- and 16-ploid Meth-A cells. Several studies have reported a constant duration of the $\mathrm{S}$ phase, regardless of the polyploidization of cultured cells (Graves and McMillan 1984, Usui et al. 1991, Brenneisen et al. 1994, Watters et al. 1994, Jordan et al. 1996, Zhang et al. 1996, Fujikawa-Yamamoto et al. 1997). Though polyploid cells are not major cell populations in mammals, our results support that an increase in ploidy and cell volume does not impair cell survival.

It should be mentioned that the DNA content of 12- and 16-ploid Meth-A cells decreased gradually. Chromosome instability at higher ploidy is well known and in some cases, the terminal ploidy was pseudo triploid (Moor et al. 1968) or aneuploid (Graves and McMillan 1984). Therefore in this study, we finished the cell-culture experiments within 2 months from the production.

\section{Acknowledgments}

This study was supported in part by a Grant-in-Aid for Scientific Research from the Ministry of Education, Culture, Sports, Science and Technology of Japan (13670228).

\section{References}

Brenneisen, P., Gogol, J. and Bayreuther, K. 1994. DNA synthesis and Fos and Jun protein expression in mitotic and postmitotic WI-38 fibroblasts in vitro. Exp. Cell Res. 211: 219-230.

Fallon, R. J. 1990. Staurosporine inhibits a tyrosine protein kinase in human hepatoma cells membranes. Biochem. Biophys. Res. Commun. 170: 1191-1196.

Fogt, F. and Nanji, A. A. 1996. Alterations in nuclear ploidy and cell phase distribution of rat liver cells in experimental alcoholic liver disease; relationship to antioxidant enzyme gene expression. Toxicol. Appl. Pharmacol. 136: 87-93.

Fried, J. 1977. Analysis of deoxyribonucleic acid histograms from flowcytofluorometry. Estimation of distribution of cells within S phase. J. Histochem. Cytochem. 25: 942-951.

Fujikawa-Yamamoto, K., Zong, Z., Murakami, M., Odashima, S., Ikeda, T. and Yoshitake, Y. 1997a. Spontaneous polyploidization results in apoptosis in a Meth-A tumor cell line. Cell Struct. Funct. 22: 399-405.

-,,- - and - 1997b. Different manner of DNA synthesis in polyploidizations of Meth-A and B16F10 cell lines. Cell Struct. Funct. 22: 527-532.

- 1999. Cell cycle analysis of DNA histograms in logarithmic scale. Cytometry Res. 9: 73-84.

-, Ohdoi, C., Yamagishi, H., Zong, Z. and Wang, S. 2000. Lectin binding in Meth-A cells polyploidized by different mechanisms. Cytologia 65: 389-395.

-, Wang, S., Yamagishi, H., Ohdoi, C., Murano, H. and Ikeda, T. 2001. Establishment of a tetraploid Meth-A cell line through polyploidization by demecolcine but not by staurosporine, K-252a and paclitaxel. Cell Prolif. 34: $211-222$.

-, Yamagishi, H. and Miyagoshi, M. 2002. Establishment of a triploid V79 cell line from tetraploid cells obtained through polyploidization using K-252a. Cell Prolif. 35: 369-379.

-, - and - 2003a. Octaploid Meth-A cells are established from a highly polyploidized cell population. Cell Prolif. 36: $87-100$.

-, Miyagoshi, M. and Yamagishi, H. 2003b. Effect of hydrochloric acid on the proliferation of tetraploid Meth-A cells. Cytometry Res. 13: 19-24.

Graves, J. A. M. and McMillan, J. 1984. Control of DNA synthesis in polyploid mammalian cells. J. Cell. Physiol. 121: $409-414$.

Jordan, M. A., Wendell, K., Gardiner, S., Derry, W. B., Copp, H. and Wilson, L. 1996. Mitotic block induced in HeLa cells by low concentrations of paclitaxel (Taxol) results in abnormal mitotic exit and apoptotic cell death. Cancer Res. 56: $816-825$.

Krishan, A. 1975. Rapid flow cytofluorometric analysis of mammalian cell cycle by propidium iodide staining. J. Cell Biol. 66: 188-193. 
Moore, J., Kieler, J. and Biczowa, B. 1968. Comparative studies of a near-tetraploid and a near-diploid line of Ehrlich's ascites tumor propagated in vivo and in vitro II, cytology and transplantability. Eur. J. Cancer 4: 81-95.

Mossin, L., Blankson, H., Huitfeldt, H. and Segren, P. O. 1994. Ploidy-dependent growth and binucleation in cultured rat hepatocytes. Exp. Cell Res. 214: 551-560.

Seglen, P. O. 1997. DNA ploidy and autophagic protein degradation as determinants of hepatocellular growth and survival. Cell Biol. Toxocol. 13: 301-315.

Tamaoki, T., Nomoto, H., Takahashi, I., Kato, Y., Morimoto, M. and Tomita, F. 1986. Staurosporine, a potent inhibitor of phospholipid/Ca ${ }^{++}$dependent protein kinase. Biochem. Biophys. Res. Commun. 135: 397-402.

Usui, T., Yoshida, M., Abe, K., Osada, M., Isono, K. and Beppu, T. 1991. Uncoupled cell cycle without mitosis induced by a protein kinase inhibitor, K-252a. J. Cell Biol. 115: 1275-1282.

Watanabe, I. and Okada, S. 1967. Effects of temperature on growth rate of cultured mammalian cells (L1578Y). J. Cell Biol. 32: 309-323.

Watters, D. J., Beamish, H. J., Marshall, K. A., Gardiner, R. A., Seymour, G. J. and Lavin, M. F. 1994. Accumulation of HL60 leukemia cells in G2/M and inhibition of cytokinesis caused by two marine compounds, bistraten A and cycloxazoline. Cancer Chemother. Pharmacol. 33: 399-409.

Zhang, Y., Wang, Z. and Ravid, K. 1996. The cell cycle in polyploid megakaryocytes is associated with reduced activity of cyclin B1-dependent cdc2 kinase. J. Biol. Chem. 271: 4266-4272.

Zollner, H. 1993. Handbook of Enzyme Inhibitors, 2nd Edn., VCH Verlagsgesellschaft mbH, Weinheim.

Zong, Z., Fujikawa-Yamamoto, K., Teraoka, K., Yamagishi, H., Tanino, M. and Odashima, S. 1994. Potentiation of K-252a, a protein kinase inhibitor-induced polyploidization, by cAMP in cultured fibrosarcoma cell line. Biochem. Biophys. Res. Commun. 205: 746-750.

—, - Tanino, M., Yamagishi, H., Gai, X. and Odashima, S. 1995. The important role of PKC in controlling polyploidy formation in cultured fibrosarcoma cell line. Biochem. Mol. Biol. Intern. 35: 1009-1015.

—, - Ota, T., Murakami, M., Li, A., Yamaguchi, N., Tanino, M. and Odashima, S. 1998. Apoptotic cell death of high polyploid cells in a cultured sarcoma cell line. Cell Struct. Funct. 23: 231-237.

_, - , Li, A., Yamaguchi, N., Chang, Y., Murakami, M. and Ishikawa, Y. 2000. Involvement of protein kinase C in taxol-induced polyploidization in a cultured sarcoma cell line. Eur. J. Pharmacol. 394: 181-187. 